\title{
Case 8: Cerebral Circulatory Arrest
}

\author{
Rita Bertuetti, Maurizio Saini, Davide Savo, \\ Francesca Simonassi, Kartika Chandrapatham, \\ and Tarek Senussi
}

49-Year-old lady, rescued at home for loss of consciousness, was found by the ambulance team in cardiac arrest (no flow time of $13 \mathrm{~min}$ ) with pulseless activity (PEA) rhythm, ACLS (advanced cardiac life support) was started, and ROSC (return of spontaneous circulation) was achieved after 17 min since the event -4 min after the arrival of the rescue team. Once in A\&E, since neither the ECG nor the ECHO showed signs of myocardial ischemia; a brain CT angio was performed; this showed aneurysmal subarachnoid hemorrhage (aSAH) Fisher 3 grade, aneurysm of the internal carotid artery, sulcal and basal cistern effacement, and signs of cerebral hypoperfusion. The patient was then admitted to ICU, where a neurological evaluation was attempted after stopping sedation:

\section{R. Bertuetti $(\bowtie)$}

Department of Anesthesiology, Critical Care Medicine and Emergency, Division of Neurocritical Care, ASST Spedali Civili di Brescia, University Hospital, Brescia, Italy

\section{Saini · D. Savo}

Department of Emergency, Perioperative Medicine and Intensive Care, Neuroanesthesia and Neurointensive Care Unit, San Gerardo Hospital, ASST-Monza, Monza, Italy

\section{F. Simonassi $\cdot$ K. Chandrapatham}

Anesthesia and Intensive Care, Ospedale Policlinico San Martino - IRCCS for Oncology and

Neurosciences, Genoa, Italy

\section{T. Senussi}

Department of Surgical Sciences and Integrated Diagnostics, University of Genoa, Genoa, Italy
GCS 3 with dilated pupils; simultanously, TCCD showed oscillatory reverse flow consistent with cerebral circulatory arrest (Fig. 32.1).

Since the clinical course (prolonged no-flow time, poor neurology), the brain CT showing brain swelling, and the TCCD proving cerebral circulatory arrest the patient was deemed unsalvageable. After few hours later the medical board for the diagnosis of brain death was summoned, and after the 6-h observation time according to the Italian law, organ donation was performed.

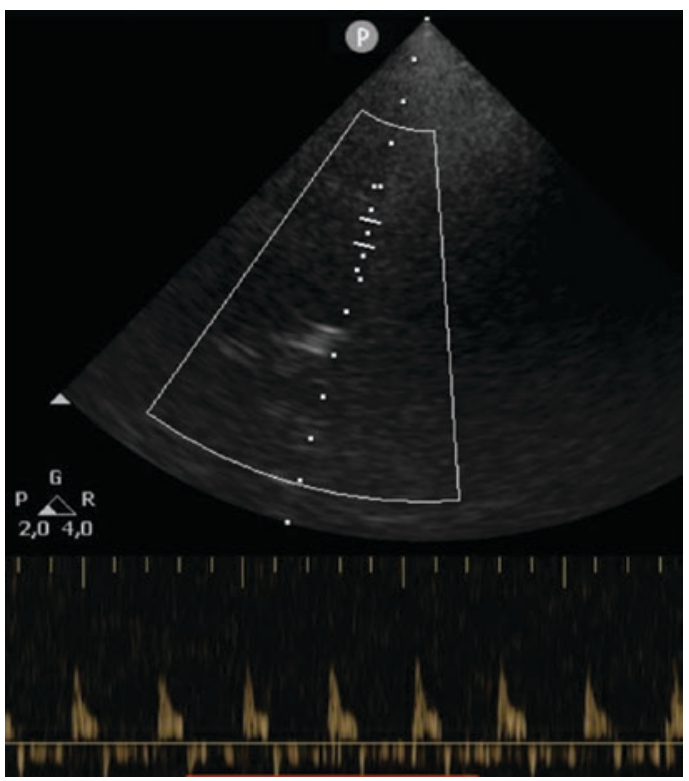

Fig. 32.1 Reverse oscillatory flow at MCA TCCD insonation 